\title{
ERRATUM
}

\author{
Daniel S. Weile • David A. Hopkins - George A. Gazonas • \\ Brian M. Powers
}

\section{Erratum to: On the proper formulation of Maxwellian electrodynamics for continuum mechanics}

Published online: 25 July 2014

(C) Springer-Verlag Berlin Heidelberg 2015

Erratum to: Continuum Mech. Thermodyn. (2014) 26:387-401 DOI 10.1007/s00161-013-0308-7

In the original publication of the article, Maxwell's equations for continuum mechanics contain a few errors of sign and indicial notation. These corrections do little to alter the conclusions of the paper except that the resultant equations are more symmetric.

Equation 8 has a small typographical error and should be

$$
\tilde{g}_{\tilde{i} \tilde{k}} \doteq \tilde{\mathrm{g}}_{i} \tilde{\mathrm{g}}_{\tilde{k}}-\tilde{\mathrm{g}}_{\tilde{i} \tilde{k}}
$$

Equation 17(b) has a slightly more significant mistake in its treatment of the metric element, and should read

$$
\tilde{\mathrm{m}}^{\tilde{i} \tilde{k}}=-\frac{\epsilon^{\tilde{\tilde{k}} \tilde{\ell}} \tilde{h}_{\tilde{\ell}}}{\sqrt{-\tilde{\mathrm{g}}}} .
$$

In Eq. 19, the order of $\tilde{\alpha}$ and $\tilde{\beta}$ are reversed, and the argument should be $m^{\tilde{\beta} \tilde{\alpha}}$. In Eq. 21, there is a spurious minus sign in $\sqrt{\tilde{g}}$. The corrected expression is

$$
\frac{1}{\sqrt{\tilde{g}}}\left[\epsilon^{\tilde{i} \tilde{k} \tilde{\ell}} \frac{\partial \tilde{h}_{\tilde{\ell}}}{\partial \tilde{x}^{\tilde{k}}}-\frac{\partial}{\partial \tilde{t}}\left(\sqrt{\tilde{g}} \tilde{d}^{\tilde{i}}\right)\right]=\tilde{j}^{\tilde{i}} .
$$

The online version of the original article can be found under doi:10.1007/s00161-013-0308-7.

D. S. Weile $(\varangle)$

Department of Electrical and Computer Engineering, University of Delaware, Newark, DE 19716, USA

E-mail:weile@udel.edu; weile@ee.udel.edu

D. A. Hopkins · G. A. Gazonas · B. M. Powers

RDRL-WMM-B Army Research Laboratory, APG, Aberdeen, MD 21005, USA

E-mail: david.a.hopkins.civ@mail.mil

G. A. Gazonas

E-mail: george.a.gazonas.civ@mail.mil

B. M. Powers

E-mail: brian.m.powers.civ@ mail.mil 
In Eq. 22, tildes are missing on some of the $\alpha$ and $\beta$ indices. The order should also be reversed to agree with the revised Eq. 19. A tilde on $\alpha$ is missing on $\tilde{j}^{\tilde{\alpha}}$ in the line after Eq. 22. A tilde is missing, and a spurious factor of $c$ appears in Eq. 30. The corrected version is

$$
\tilde{v}^{\tilde{i}} \doteq c \frac{\mathrm{d} \tilde{\mathbf{X}}^{\tilde{i}}}{\mathrm{~d} \tilde{\mathbf{X}}^{0}}=-\frac{\partial \tilde{x}^{\tilde{i}}}{\partial x^{i}} v^{i}
$$

A minus sign is missing in the $\tilde{g}_{i \tilde{k}}$ term in Eq. 34. The equation should be

$$
\tilde{\mathrm{g}}_{\tilde{i} \tilde{k}}=-\delta_{i k} \frac{\partial x^{i}}{\partial \tilde{x}^{\tilde{i}}} \frac{\partial x^{k}}{\partial \tilde{x}^{\tilde{k}}}
$$

Unlike the previous errors, this one carries into the remainder of the paper, causing errors in later expressions. The right Cauchy-Green tensor $C_{I K}$ expression, Eq. 35, should then be

$$
\tilde{\mathrm{g}}_{\tilde{i} \tilde{k}}=-\delta_{i k} \frac{\partial x^{i}}{\partial \tilde{x}^{\tilde{i}}} \frac{\partial x^{k}}{\partial \tilde{x}^{\tilde{k}}}=-\delta_{\tilde{i}}^{I} \delta_{\tilde{k}}^{K} \delta_{i k} \frac{\partial x^{i}}{\partial X^{I}} \frac{\partial x^{k}}{\partial X^{K}}=-\delta_{\tilde{i}}^{I} \delta_{\tilde{k}}^{K} C_{I K},
$$

and Eq. 36 is therefore

$$
\tilde{\mathbf{g}}^{00}=1, \quad \tilde{\mathbf{g}}^{\tilde{i} 0}=\tilde{\mathbf{g}}^{0 \tilde{i}}=\frac{\tilde{v}^{\tilde{i}}}{c}, \quad \tilde{\mathbf{g}}^{\tilde{i} \tilde{k}}=-\delta^{i k} \frac{\partial \tilde{x}^{\tilde{i}}}{\partial x^{i}} \frac{\partial \tilde{x}^{\tilde{k}}}{\partial x^{k}}
$$

In Eq. 37, the derivatives are with respect to the four-vector $\tilde{\mathbf{x}}^{\tilde{\alpha}}$, not the purely spatial components $\tilde{x}^{i}$ and should therefore be

$$
\tilde{\mathrm{n}}_{\tilde{\alpha} \tilde{\beta}}=\frac{\partial x^{\alpha}}{\partial \tilde{\mathbf{x}}^{\tilde{\alpha}}} \frac{\partial x^{\beta}}{\partial \tilde{\mathbf{x}}^{\tilde{\beta}}} \mathrm{n}_{\alpha \beta}
$$

Equation 52 is obviously incorrect as the units do not match. It should read

$$
\tilde{\mathbf{x}}^{0}=\tilde{a} \mathbf{x}^{0}+\tilde{s_{i}} \tilde{\mathbf{x}}^{\tilde{i}} .
$$

A sign error appears in Eq. 54, and is carried to other equations. The corrected expressions are

$$
\frac{\partial \mathbf{x}^{0}}{\partial \tilde{\mathbf{x}}^{0}}=\frac{1}{\tilde{a}}, \quad \frac{\partial \mathbf{x}^{0}}{\partial \tilde{\mathbf{x}}^{\tilde{i}}}=-\frac{\tilde{s}_{\tilde{i}}}{\tilde{a}}, \quad \frac{\partial \mathbf{x}^{i}}{\partial \tilde{\mathbf{x}}^{0}}=\frac{1}{\tilde{a}} \frac{v^{i}}{c}, \quad \frac{\partial \mathbf{x}^{i}}{\partial \tilde{\mathbf{x}}^{\tilde{i}}}=\delta_{\tilde{i}}^{I} \frac{\partial x^{i}}{\partial X^{I}}-\frac{\tilde{s_{\tilde{i}}}}{\tilde{a}} \frac{v^{i}}{c} .
$$

Incorporating these changes and correcting for an erroneous square root, Eq. 55 should read

$$
\tilde{s}_{\tilde{i}}=-\frac{\tilde{a} \delta_{i j} \frac{\partial x^{j}}{\partial \tilde{x}^{\tilde{i}}} \frac{v^{i}}{c}}{1-\delta_{k \ell} \frac{v^{k} v^{\ell}}{c^{2}}} .
$$

The sign error propagates further to Eq. 58 which should now be

$$
\frac{\partial \tilde{\mathbf{x}}^{0}}{\partial \mathbf{x}^{0}}=1, \quad \frac{\partial \tilde{\mathbf{x}}^{0}}{\partial \mathbf{x}^{i}}=-\delta_{i j} \frac{v^{j}}{c}, \quad \frac{\partial \tilde{\mathbf{x}}^{\tilde{i}}}{\partial \mathbf{x}^{0}}=\frac{\tilde{v}^{\tilde{i}}}{c}, \quad \frac{\partial \tilde{\mathbf{x}}^{\tilde{i}}}{\partial \mathbf{x}^{i}}=\frac{\partial \tilde{x}^{\tilde{i}}}{\partial x^{i}} .
$$

In Eq. 60 , the $\tilde{g}_{i} \tilde{j}$ term should be

$$
\tilde{\mathrm{g}}_{i \tilde{j}}=-\tilde{g}_{\tilde{i} \tilde{j}}=-\delta_{i j} \frac{\partial x^{i}}{\partial \tilde{x}^{\tilde{i}}} \frac{\partial x^{j}}{\partial \tilde{x}^{\tilde{j}}},
$$

as the expression presented in the paper is obviously not a proper indicial expression. In Eq. 61, the indices on $\tilde{g}^{\tilde{i} \tilde{j}}$ should be raised. The sign corrections lead to changes in the definitions given by Eqs. 62-64. The corrected definitions are

$$
\begin{aligned}
& \tilde{e}^{\tilde{i}} \doteq \tilde{g}^{\tilde{i} \tilde{j}} \tilde{e}_{\tilde{j}}, \quad \tilde{d}_{\tilde{i}} \doteq \tilde{g}_{\tilde{i} \tilde{j}} \tilde{d}^{\tilde{j}}, \\
& \tilde{h}^{\tilde{i}} \doteq \tilde{g}^{\tilde{j}} \tilde{h}_{\tilde{j}}, \quad \tilde{b}_{\tilde{i}} \doteq \tilde{g}_{\tilde{i} \tilde{j}} \tilde{b}^{\tilde{j}}, \\
& \tilde{p}_{\tilde{i}} \doteq \tilde{g}_{\tilde{i} \tilde{j}} \tilde{p}^{\tilde{j}}, \quad \tilde{m}^{\tilde{i}} \doteq \tilde{g}^{\tilde{j}} \tilde{m}_{\tilde{m}} \text {. }
\end{aligned}
$$


Erratum to: On the Proper Formulation of Maxwellian...

Equations 66-67 now become

$$
\begin{array}{ll}
\tilde{m}_{\tilde{i} 0}=-c \tilde{d}_{\tilde{i}}, & \tilde{m}_{\tilde{i} \tilde{j}}=-\sqrt{\tilde{g}} \epsilon_{\tilde{i} \tilde{j} \tilde{k}} \tilde{h}^{\tilde{k}}, \\
\tilde{p}_{\tilde{i} 0}=c \tilde{p}_{\tilde{i}}, & \tilde{p}_{\tilde{i} \tilde{j}}=-\sqrt{\tilde{g}} \epsilon_{\tilde{i} \tilde{j} \tilde{k}} \tilde{m}^{\tilde{k}} .
\end{array}
$$

The sign error then propagates to Eqs. 68-73, which become

$$
\begin{aligned}
& \tilde{e}_{\tilde{i}}=\frac{\partial x^{i}}{\partial \tilde{x}^{\tilde{i}}}\left(e_{i}+\epsilon_{i j k} v^{j} b^{k}\right), \quad \tilde{b}^{\tilde{i}}=\frac{\partial \tilde{x}^{\tilde{i}}}{\partial x^{i}}\left(b^{i}-\epsilon^{i j k} \frac{v_{j} e_{k}}{c^{2}}\right), \\
& \tilde{e}^{\tilde{i}}=\frac{\partial \tilde{x}^{\tilde{i}}}{\partial x^{i}}\left(e^{i}+\epsilon^{i j k} v_{j} b_{k}\right), \quad \tilde{b}_{\tilde{i}}=\frac{\partial x^{i}}{\partial \tilde{x}^{\tilde{i}}}\left(b_{i}-\epsilon_{i j k} \frac{v^{j} e^{k}}{c^{2}}\right), \\
& \tilde{d}^{\tilde{i}}=\frac{\partial \tilde{x}^{\tilde{i}}}{\partial x^{i}}\left(d^{i}+\epsilon^{i j k} \frac{v_{j} h_{k}}{c^{2}}\right), \quad \tilde{h}_{\tilde{i}}=\frac{\partial x^{i}}{\partial \tilde{x}^{\tilde{i}}}\left(h_{i}-\epsilon_{i j k} v^{j} d^{k}\right), \\
& \tilde{p}^{\tilde{i}}=\frac{\partial \tilde{x}^{\tilde{i}}}{\partial x^{i}}\left(p^{i}-\epsilon^{i j k} \frac{v_{j} m_{k}}{c^{2}}\right), \quad \tilde{m}_{\tilde{i}}=\frac{\partial x^{i}}{\partial \tilde{x}^{\tilde{i}}}\left(m_{i}+\epsilon_{i j k} v^{j} p^{k}\right), \\
& p^{i}=\frac{\partial x^{i}}{\partial \tilde{x}^{\tilde{i}}}\left(\tilde{p}^{\tilde{i}}-\frac{\epsilon^{\tilde{i} \tilde{j} \tilde{k}}}{\sqrt{\tilde{g}}} \frac{\tilde{v}_{\tilde{j}} \tilde{m}_{\tilde{k}}}{c^{2}}\right), \quad m_{i}=\frac{\partial \tilde{x}^{\tilde{i}}}{\partial x^{i}}\left(\tilde{m}_{\tilde{i}}+\sqrt{\tilde{g}} \epsilon_{\tilde{i} \tilde{j} \tilde{k}} \tilde{v}^{\tilde{j}} \tilde{p}^{\tilde{k}}\right), \\
& \tilde{\rho}=\rho-\frac{v_{i} j^{i}}{c^{2}}, \quad \tilde{j}^{\tilde{i}}=\frac{\partial \tilde{x}^{\tilde{i}}}{\partial x^{i}}\left(j^{i}-\rho v^{i}\right) .
\end{aligned}
$$

Finally, the corrected versions of Tables 1, 2 and 3 are shown. As can be seen, after these corrections for sign errors, the time orthogonal transformation equations exhibit a completely symmetric relationship. They are also in complete agreement with the well-known frame transformation laws for electromagnetic field quantities in the limit of rigid motion. In contrast, even with the corrections, the transformation rules commonly used in continuum mechanics [1-4] remain asymmetric. As mentioned in the paper, this asymmetry is a source of confusion and possible errors in formulations that rely on them.

Table 1 Transformation of the elements of the electromagnetic tensor in different formulations

\begin{tabular}{lll}
\hline & $\mathbf{e}$ & $\mathbf{b}$ \\
\hline Universal time, $\tilde{n}_{\alpha \beta}$ & $\tilde{e}_{\tilde{i}}=\frac{\partial x^{i}}{\partial \tilde{x}_{\tilde{\tilde{I}}}}\left(e_{i}+\epsilon_{i j k} v^{j} b^{k}\right)$ & $\tilde{b}^{\tilde{i}}=\frac{\partial \tilde{x}^{\tilde{i}}}{\partial x^{i}} b^{i}$ \\
Universal time, $\tilde{n}^{\alpha \beta}$ & $\tilde{e}^{\tilde{i}}=\frac{\partial \tilde{x}_{i}^{i}}{\partial x^{i}} e^{i}$ & $\tilde{b}_{\tilde{i}}=\frac{\partial x^{i}}{\partial \tilde{x}_{\tilde{i}}^{i}}\left(b_{i}-\epsilon_{i j k} \frac{v^{j} e^{k}}{c^{2}}\right)$ \\
Time orthogonal, $\tilde{n}_{\alpha \beta}$ & $\tilde{e}_{\tilde{i}}=\frac{\partial x^{i}}{\partial \tilde{x}^{\tilde{i}}}\left(e_{i}+\epsilon_{i j k} v^{j} b^{k}\right)$ & $\tilde{b}^{\tilde{i}}=\frac{\partial \tilde{x}^{\tilde{x}}}{\partial x^{i}}\left(b^{i}-\epsilon^{i j k} \frac{v_{j} e_{k}}{c^{2}}\right)$ \\
Lax and Nelson [3] & $E_{I}=\frac{\partial x_{i}}{\partial X_{I}}\left(e_{i}+\epsilon_{i j k} v_{j} b_{k}\right)$ & $B_{I}=\sqrt{C} \frac{\partial X_{I}}{\partial x_{i}} b_{i}$ \\
Yang and Batra [4] & $E_{I}=\sqrt{C} \frac{\partial X_{I}}{\partial x_{i}} e_{i}$ & (Electrostatic) \\
Dorfmann and Ogden [2] & $E_{I}=\frac{\partial x_{i}}{\partial X_{I}} e_{i}$ & (Electrostatic) \\
Clayton [1] & $E_{I}=\frac{\partial x_{i}}{\partial X_{I}} e_{i}$ & (Electrostatic) \\
\hline
\end{tabular}

Table 2 Transformation of the elements of the displacement tensor in different formulations

\begin{tabular}{lll}
\hline & $\mathbf{d}$ & $\mathbf{h}$ \\
\hline Universal time, $\tilde{m}^{\alpha \beta}$ & $\tilde{d}^{\tilde{i}}=\frac{\partial \tilde{x}^{\tilde{i}}}{\partial x^{i}} d^{i}$ & $\tilde{h}_{\tilde{i}}=\frac{\partial x^{i}}{\partial \tilde{x}_{\tilde{i}}^{i}}\left(h_{i}-\epsilon_{i j k} v^{j} d^{k}\right)$ \\
Universal time, $\tilde{m}_{\alpha \beta}$ & $\tilde{d}_{\tilde{i}}=\frac{\partial x^{i}}{\partial \tilde{x}^{\tilde{i}}}\left(d_{i}+\epsilon_{i j k} \frac{v^{j} h^{k}}{c^{2}}\right)$ & $\tilde{h}^{\tilde{i}}=\frac{\partial \tilde{x}_{\hat{i}}}{\partial x^{i}} h^{i}$ \\
Time orthogonal, $\tilde{m}^{\alpha \beta}$ & $\tilde{d}^{\tilde{i}}=\frac{\partial \tilde{x}^{i}}{\partial x^{i}}\left(d^{i}+\epsilon^{i j k} \frac{v_{j} h_{k}}{c^{2}}\right)$ & $\tilde{h}_{\tilde{i}}=\frac{\partial x^{i}}{\partial \tilde{x}_{\tilde{i}}}\left(h_{i}-\epsilon_{i j k} v^{j} d^{k}\right)$ \\
Lax and Nelson [3] & $D_{I}=\sqrt{C} \frac{\partial X_{I}}{\partial x_{i}} d_{i}$ & $H_{I}=\frac{\partial x_{i}}{\partial X_{I}}\left(h_{i}-\epsilon_{i j k} v_{j} d_{k}\right)$ \\
Yang and Batra [4] & $D_{I}=\sqrt{C} \frac{\partial X_{I}}{\partial x_{i}} d_{i}$ & (Electrostatic) \\
Dorfmann and Ogden [2] & $D_{I}=\sqrt{C} \frac{\partial X_{I}}{\partial x_{i}} d_{i}$ & (Electrostatic) \\
Clayton [1] & $D_{I}=\sqrt{C} \frac{\partial X_{I}}{\partial x_{i}} d_{i}$ & (Electrostatic) \\
\hline
\end{tabular}


D. S. Weile et al.

Table 3 Transformation of the elements of the polarization tensor in different formulations

\begin{tabular}{lll}
\hline & $\mathbf{p}$ & $\mathbf{m}$ \\
\hline Universal time, $\tilde{p}^{\alpha \beta}$ & $\tilde{p}^{\tilde{i}}=\frac{\partial \tilde{x}^{\tilde{i}}}{\partial x^{i}} p^{i}$ & $\tilde{m}_{\tilde{i}}=\frac{\partial x^{i}}{\partial \tilde{x}_{\tilde{i}}^{i}}\left(m_{i}+\epsilon_{i j k} v^{j} p^{k}\right)$ \\
Universal time, $\tilde{p}_{\alpha \beta}$ & $\tilde{p}_{\tilde{i}}=\frac{\partial x^{i}}{\partial \tilde{x}_{\tilde{i}}}\left(p_{i}-\epsilon_{i j k} \frac{v^{j} m^{k}}{c^{2}}\right)$ & $\tilde{m}^{\tilde{i}}=\frac{\partial \tilde{x}_{i}^{i}}{\partial x^{i}} m^{i}$ \\
Time orthogonal, $\tilde{p}^{\alpha \beta}$ & $\tilde{p}^{\tilde{i}}=\frac{\partial \tilde{x}^{i}}{\partial x^{i}}\left(p^{i}-\epsilon^{i j k} \frac{v_{j} m_{k}}{c^{2}}\right)$ & $\tilde{m}_{\tilde{i}}=\frac{\partial x^{i}}{\partial \tilde{x}^{i}}\left(m_{i}+\epsilon_{i j k} v^{j} p^{k}\right)$ \\
Lax and Nelson [3] & $P_{I}=\sqrt{C} \frac{\partial X_{I}}{\partial x_{i}} p_{i}$ & $M_{I}=\frac{\partial x^{i}}{\partial X^{I}} m_{i}$ \\
Yang and Batra [4] & $P_{I}=\sqrt{C} \frac{\partial X_{I}}{\partial x_{i}} p_{i}$ & (Electrostatic) \\
Dorfmann and Ogden [2] & $P_{I}=\sqrt{C} \frac{\partial X_{I}}{\partial x_{i}} p_{i}$ & (Electrostatic) \\
Clayton [1] & $P_{I}=\frac{\partial x^{i}}{\partial X^{I}} p_{i}$ & (Electrostatic) \\
\hline
\end{tabular}

Acknowledgments The authors are grateful to Dr. Christian Celigoj for his comments concerning the sign issues with the paper.

\section{References}

1. Clayton, J.: A non-linear model for elastic dielectric crystals with mobile vacancies. Int. J. Non-Linear Mech. 44(6), 675688 (2009)

2. Dorfmann, A.R., Ogden, R.W.: Nonlinear electroelasticity. Acta Mech. 174(3-4), 167-183 (2000)

3. Lax, M., Nelson, D.: Maxwell equations in material form. Phys. Rev. B 13(4), 1777-1784 (1976)

4. Yang, J.S., Batra, R.C.: A theory of electroded thin thermopiezoelectric plates subject to large driving voltages. J. Appl. Phys. 76(9), 5411-5417 (1994) 